\title{
Multiple Sensor Fusion for Detecting Location of Curbs, Walls, and Barriers ${ }^{1}$
}

\author{
Romuald Aufrère \\ LASMEA \\ Université Blaise-Pascal \\ Clermont-Ferrand, France \\ aufrere@lasmea.univ-bpclermont.fr
}

\author{
Christoph Mertz \\ Robotics Institute \\ Charles Thorpe \\ Carnegie Mellon University \\ Pittsburgh, Pennsylvania, USA \\ cmertz@andrew.cmu.edu_cet@cs.cmu.edu
}

\begin{abstract}
Knowledge of the location of curbs, walls, or barriers is important for guidance of vehicles or for the understanding of their surroundings. We have developed a method to detect such continous objects alongside and in front of a host vehicle. We employ a laser line striper, a vehicle state estimator, a video camera, and a laser scanner to detect the object at one location, track it alongside the vehicle, search for it in front of the vehicle and eliminate erroneous readings caused by occlusion from other objects.
\end{abstract}

\section{Introduction}

Systems for driver warning or autonomous driving need a comprehensive understanding of the vehicle environment $[9,10]$. A case in point is the detection of curbs, barriers, or other physical manifestations of the limit of the road. It is the natural extension of determining the location of the road by detecting lines to detecting physical limits. The reasons and applications for line detection, a topic of great interest in the past and present $[1,2,4,8]$, apply to the detection of physical limits of the road: lane keeping, lane departure warning, and determination if a detected object is in the same lane. The last point is of particular interest in urban settings in relation to pedestrians. E.g. in our research concerning warning systems for transit buses $[6,7]$ we found that pedestrian are often in close proximity to the bus, but as long as they are on the sidewalk, the situation can be considered safe. The position of the curb is therefore an important piece of information to determine the level of danger in a situation involving pedestrian.

\footnotetext{
1 This work was partially sponsored by PennDOT under agreement \# 62N111, project TA-34, funded by the USDOT Federal Transit Administration, and prepared through collaborative participation in the Robotics Consortium sponsored by the U. S. Army Research Laboratory under the Collaborative Technology Alliance Program, Cooperative Agreement DAAD19-01-2-0012. The U. S. Government is authorized to reproduce and distribute reprints for Government purposes notwithstanding any copyright notation thereon.
}

The work described in this article is mainly focused on curb detection, but applies similarly to detection of barriers, walls, etc. The output of various sensors is fused to obtain the curb position along and in front of the vehicle. Our process to detect the curb is mainly based on three different modules. The first one permits an accurate detection and location of the curb at one place. The second uses the information of the vehicle state to track the curb position alongside the vehicle. This track is used in the last step of our process to initialize a search of the curb in a video image, which then gives a track of the curb in front of the vehicle.

\section{Our approach}

In order to be useful for automotive purposes, the system needed to be developed under following constraints:

1. low cost and a small size

2. robust detection

3. effective under most weather conditions

4. real time detection

5. possibility of collaboration with other modules

Taking into account these constraints, we used three modules, which facilitated four different sensors. The sensors can be seen mounted on our experimental vehicle NAVLAB 11 Figure 1.

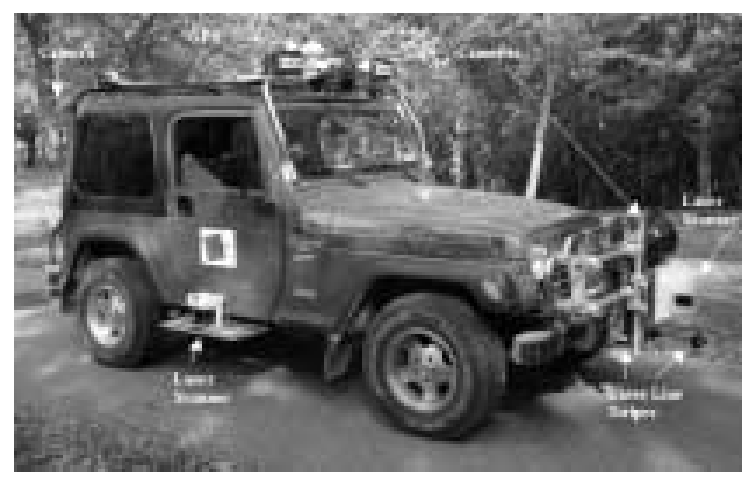

Figure 1: The experimental vehicle NAVLAB11 as a platform for various sensors. 
In the front are a laser and a camera which form the laser line striper. Low on the right side is a $\operatorname{SICK}^{\circledR}$ laser scanner and on the right back corner of the roof is a camera. The vehicle state estimator consists of an IMU and wheel encoder.

\subsection{Curb Detection}

The main goal of this module is to accurately determine the distance from the vehicle to the curb at one location. Besides having the characteristics mentioned above, the sensor should have a high resolution (few $\%$ in distance, $<$ $1^{\circ}$ in angle) and an operating range of up to $3 \mathrm{~m}$. For this purpose we built a laser triangulation sensor. The detailed description of this laser line striper is presented in [5], here we give a short summary.

\subsubsection{Laser line striper}

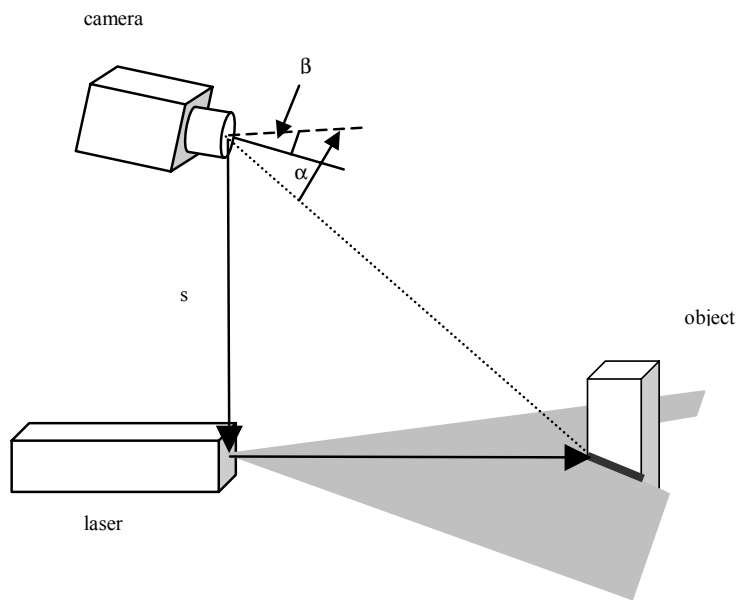

Figure 2: Geometric configuration of a laser line striper.

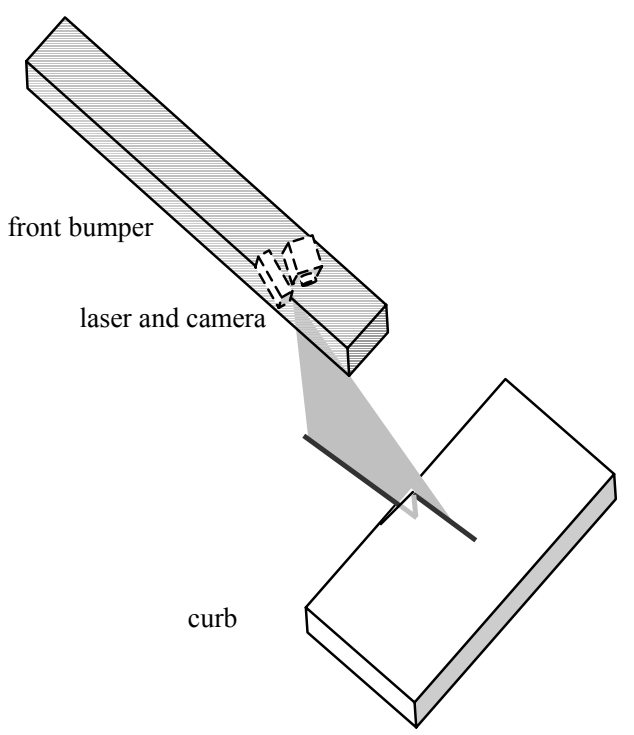

Figure 3: Schematic of the curb detector.

The light of a laser is fanned out to form a plane of light (see Figure 2). If an object intersects the plane, the reflected light is observed by a camera placed at a distance from the laser. The distance between laser and object can be determined by triangulation:

$$
d=\operatorname{s.cot}(\alpha)
$$

where $s$ is the camera-laser distance and $\alpha$ the angle at which the camera sees the object (see Figure 2).

To make a system work outside, where the sun produces a strong background of light, we employ two techniques to reduce the background. In the one hand, the camera is equipped with a narrow filter and in the other hand the laser is pulsed and in synchronization with the fast shutter of the camera.

For the purpose of curb detection the laser line striper is placed beneath the front bumper of the vehicle looking to the side. A schematic of the arrangement is shown in Figure 3.

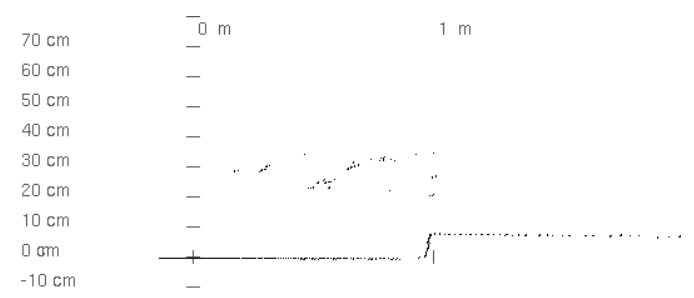

Figure 4: Profile of the road and curb observed by the laser line striper. Some erroneous readings can be seen above the road.

An example of a single measurement of the road and curb profile can be seen in Figure 4 Both can be clearly seen, but also some egregious readings are present. Those are caused by reflectance of sunlight, which can sometimes be stronger than the reflectance of the laser. Those errors will be greatly reduced in a future version of the sensor. But the histogram algorithm, which extracts the curb position from the profile, is already insensitive to such noise.

\subsubsection{Histogram}

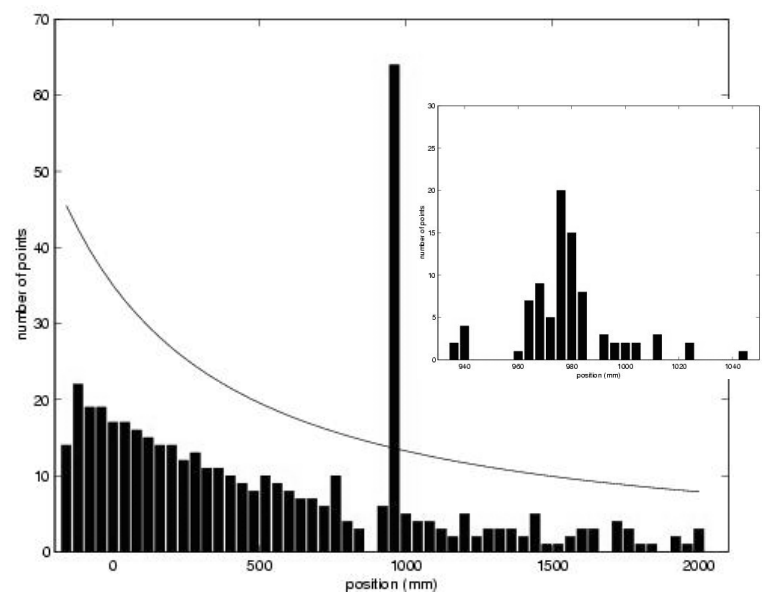

Figure 5: Histogram of the number of data points versus horizontal distance. The black line is the detection threshold. The insert shows the histogram around the peak in an increased resolution. 
The output data of the laser line striper is accumulated in a histogram along the horizontal axis with a predetermined bin size, in our case, $4 \mathrm{~cm}$. The histogram of the data presented in Figure 4 is shown in Figure 5 The greatest bin with an accumulation greater than a threshold is considered a curb. The threshold is indicated in Figure 5 as a black line. The threshold value is set to:

$$
N_{t}=a /\left(x+x_{0}\right)^{2}+b /\left(x+x_{0}\right)
$$

$x_{0}$ is the point where the camera is perpendicular to the laser plane. The first term is the number of points per bin expected if no curb is present. In our example $a=17$. The second term is the additional number of points required. The parameter $b$ corresponds to one minimum curb height independent of the distance $\mathrm{x} . \quad b$ can be adjusted, trading off detection sensitivity and noise susceptibility. From the data around the bin identified as the curb another histogram is formed, now with a much smaller bin size of $4 \mathrm{~mm}$ (insert in Figure 5. The weighted average of the positions of the maximum bin and its two adjunct bins is calculated and is the final location of the curb.

The computational time and, more importantly, the robustness of the detection can be improved, if estimates of the curb position of previous measurements are used. We use earlier measurements of the curb position in front of the vehicle (see Section 2.3 to roughly predict the current curb position on the side. The above mentioned histogram method is then only applied to the data around the predicted position.

\subsection{Curb Tracking}

Our test vehicle NavLab11 is equipped with an odometer, an IMU, and a GPS. This collection of sensors provides the vehicle state, namely position, speed, and heading. Knowledge of the current and past vehicle state allows for the transformation of past curb measurements into the current frame of reference. It is therefore possible to track the curb alongside the vehicle. Figure 6 shows an example of a track.

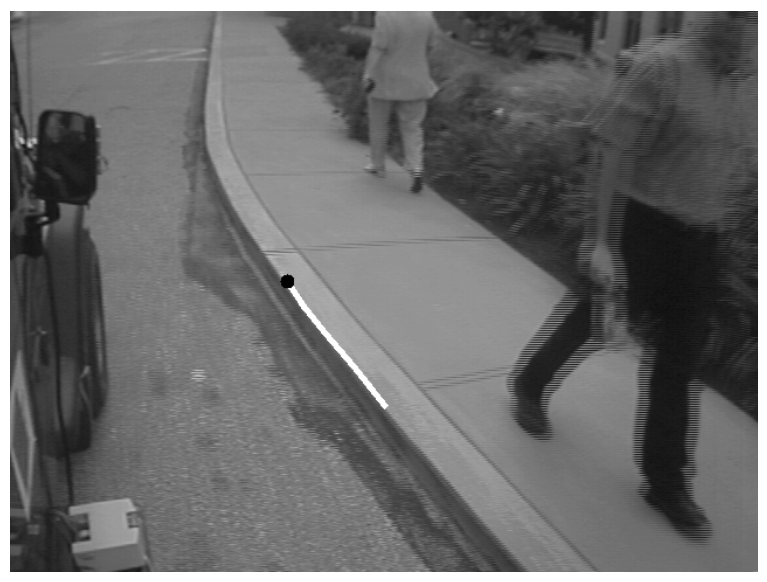

Figure 6: Current detection (black circle) and track (white line) of the curb viewed by a camera mounted on the side of the vehicle.
Assuming the vehicle is driving forward, detection and tracking can only provide the position of the curb alongside or behind the vehicle. In order to see the curb ahead one needs to employ a forward-looking sensor like a camera. The track is used to initialize a search of the curb in a video image.

\subsection{Forward looking Curb Detection}

A monocular monochromatic camera is mounted on the top rear right corner of the vehicle, looking down along the side of the vehicle. The video image is used to visualize the environment besides the vehicle as well as to provide the raw data for vision based curb detection. An example of the images provided by this camera is presented in Figure 6

\subsubsection{Definition of the interest zones}

The detections are realized in interest zones. To determine the first interest zone, all the track points from the previous module are transformed into the image reference frame. We use the two last points to compute the origin and slope of the curb in the image. The origin defines the lower middle point of a rectangle and the slope its orientation (Figure 7). The rectangle has a height of 40 pixels.

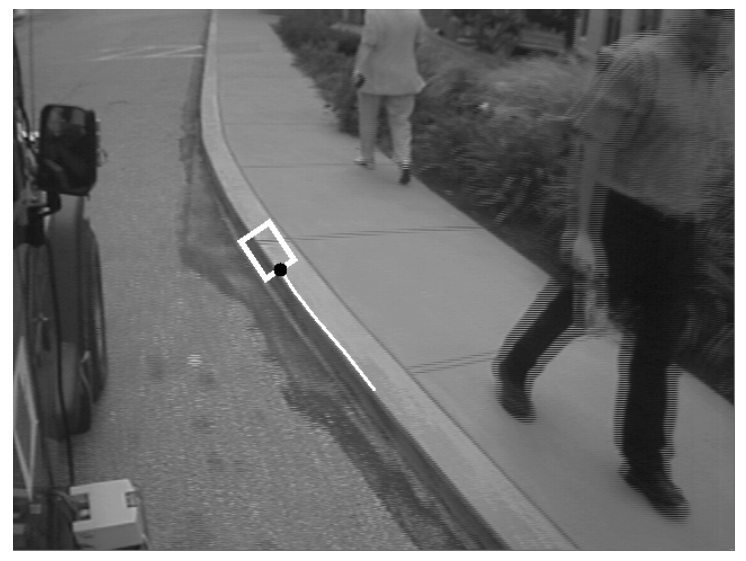

Figure 7: Interest zone (white rectangle) in the image with current curb detection (black dot) and the track (white line).

Following zones are built in the same manner, where newly determined points define the new origin and slope.

\subsubsection{Detection}

For each line inside the interest zone the point with the maximum contrast is determined. We use the canny edge detector [3] to find these points which represent the curb in the zone. If there are a sufficient number of these points, they are fitted to a straight-line segment by a median least square method.

The algorithm gives two points, the intersection between the detected segment and the upper and lower horizontal 
borders of the interest zone. After a segment is found, the process is iterated, i.e. new definition of an interest zone based on the just found segment, etc. The process stops when the number of the detected points in the last interest zone is not sufficient or if the interest zone is above a maximum height in the image. Consecutive segments represent the sidewalk's edge, an example is shown in Figure 8

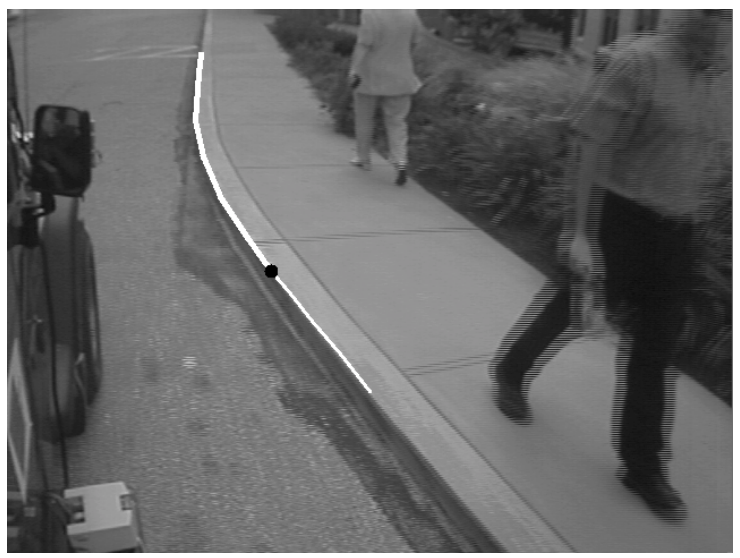

Figure 8: Detected curb alongside and in front of the vehicle.

At the end of this process a confidence score is calculated based on the number of detected points in relation to the height of the interest zone. This score is used when the laser line striper is not able to detect the curb on the side of the vehicle, e.g. when the vehicle drives along a curb ramp (Figure 9.)

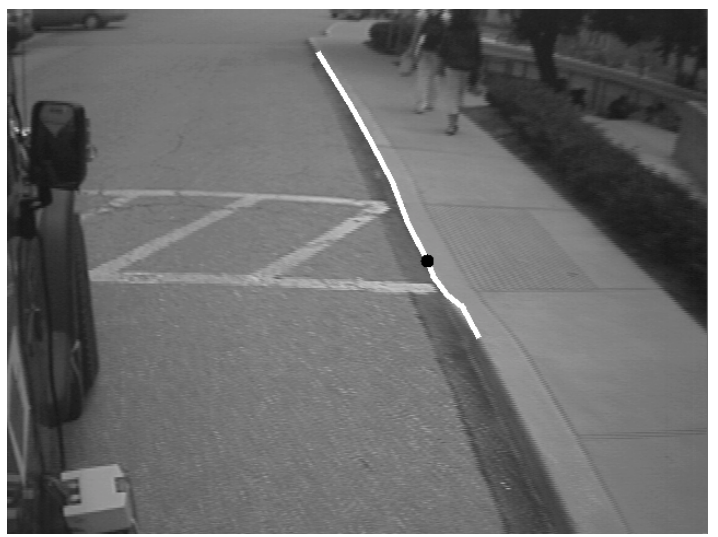

Figure 9: Curb ramp on the side of the vehicle. The laser line striper cannot see the curb along the ramp, it is followed by the video detection instead.

The line striper cannot see the curb, because it is not high enough at that location (Figure 10. If the confidence score at such a location is high enough, the location of the curb is determined by the video detection.

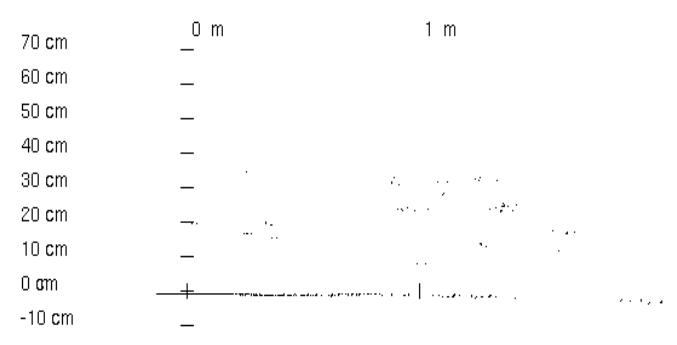

Figure 10: Profile of curb ramp seen by the line striper.

\subsubsection{Obstacles}

It was found that the curb detection with video gives false measurements when an object is close to the curb. The edge of the object can be mistaken for the edge of the curb (e.g. see Figure 11.

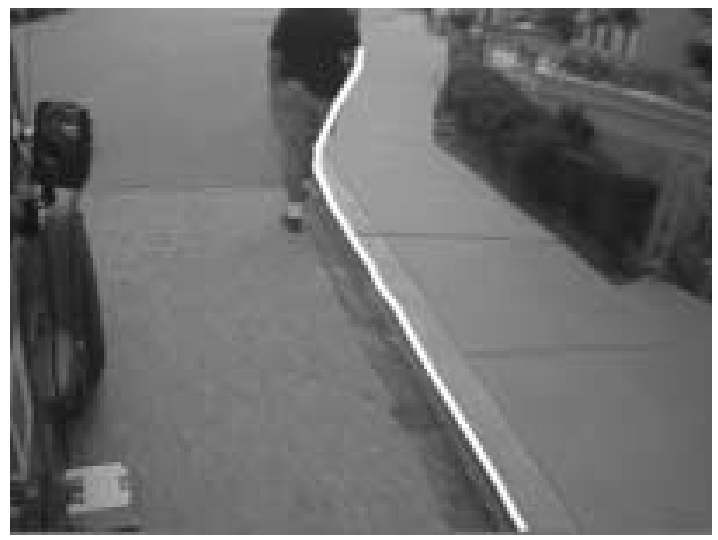

Figure 11: Erroneous curb detection caused by a pedestrian in front of the curb.

This problem can be solved if one knows that an object is present and the search can then be changed accordingly.

A SICK laser scanner gives the information of objects present in the vicinity of the vehicle. This sensor is installed on the side of the vehicle. The data provided by this sensor represent the distances of obstacles measured about $0.5 \mathrm{~m}$ off the ground. Those positions are projected unto the ground and when the search of the curb in the image comes close to these projected positions or the area above those positions, the detected points are removed. As a consequence, there won't be enough points in an interest zone that includes the object and the search is stopped.

Figure 12 shows the same situation as in Figure 11, but now the additional information of the laser scanner is provided indicated as small white circles. The detection of the curb is correctly terminated when it reaches the obstacle. 


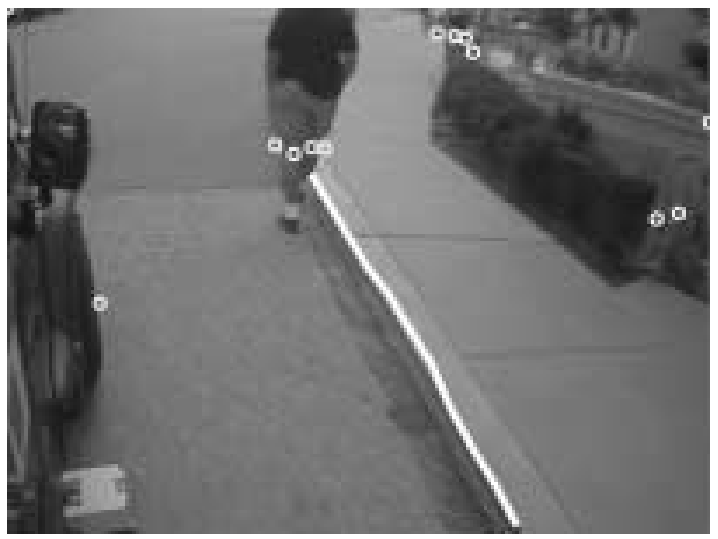

Figure 12: Video data with obstacle information. The raw image is the same as in Figure 11, the data from the laser scanner is indicated as white circles. The detection of the curb is correctly terminated when it reaches the obstacle.

\section{Further Results}

In the sections above are examples of a straight curb, a curved curb, a partially occluded curb, and a curb with a ramp. Figure 13 shows the case of a wounded curb.

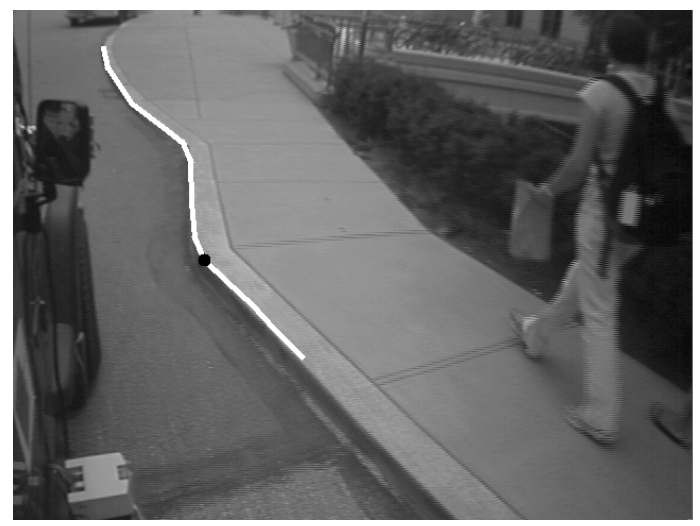

Figure 13: Detection of a wounded curb.

Our system is also able to detect the edges of a barrier (Figure 14 and walls (Figure 15 and Figure 16.

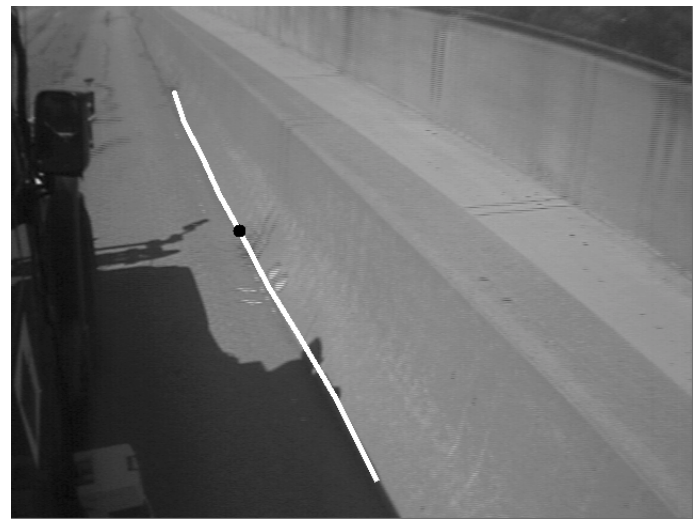

Figure 14: Detecting the edge of a barrier alongside a road.

As in the previous figures, the black point indicates the current edge detection by the laser line striper, the white line behind it is the tracked edge position using the vehicle state information and finally the white line in front of the black dot is the positions provided by the video detection.

In all three cases the edges are accurately detected. However, there is a gutter at the brick wall and a dark vertical break in the indoor wall, which can be seen in the left upper corner of the respective images (Figure 15 and Figure 16. Both of them will result in incorrect detection of the edges of the walls similarly to the object in front of the curb in Figure 11

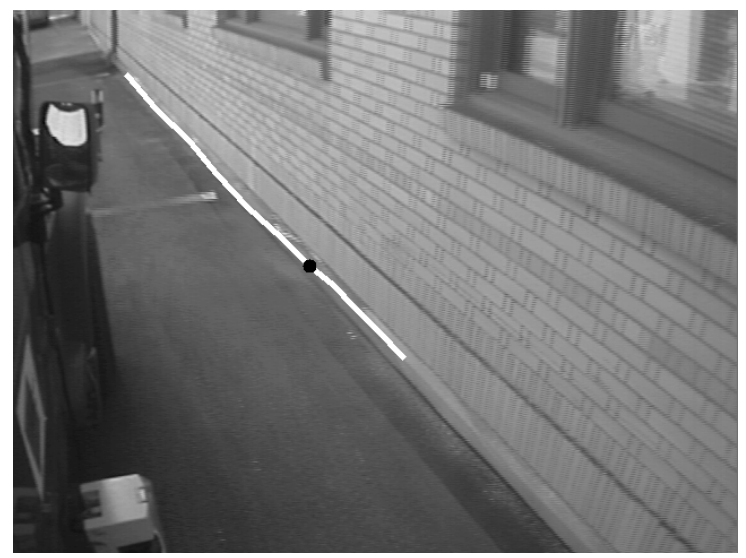

Figure 15: Detecting the edge of an outdoor brick wall.

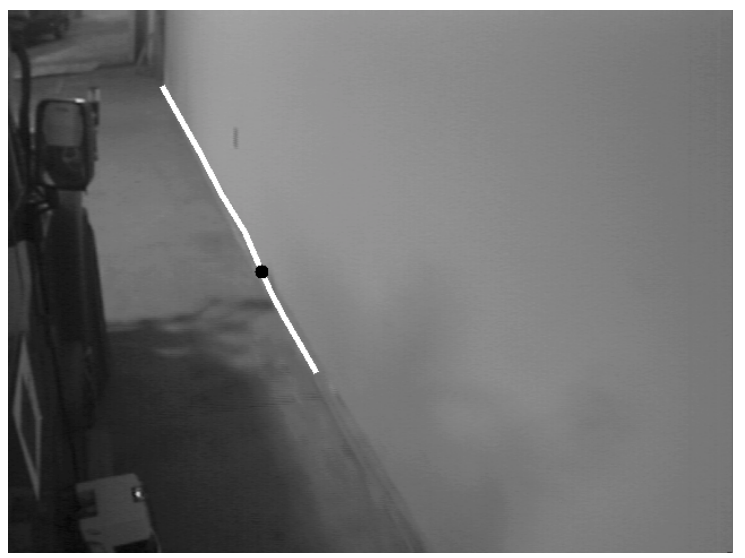

Figure 16: Detecting the edge of an indoor wall.

\section{Uncertainties}

The uncertainty of the single point distance measurements of the laser line striper depends on its configuration. The sensor used in our examples had the uncertainty characteristics shown in Figure 17 This uncertainty does not include sub-pixel resolution (for details about uncertainties see [5]). The uncertainty increases quadratically from $\pm 1 \mathrm{~mm}$ to $\pm 9 \mathrm{~mm}$ at a distance of $2 \mathrm{~m}$. The distance curb-vehicle is the result of analyzing many of the single points with the histogram method. The width 
of the peak in the $4 \mathrm{~mm}$ resolution histogram (Figure 5 indicates that the uncertainty in the curb-vehicle distance measurement is several millimeters. We measured the curb-vehicle distance uncertainty by driving along a straight curb while driving straight and comparing the measured distances. The distances were around $1 \mathrm{~m}$ and varied by a sigma of $6 \mathrm{~mm}$.

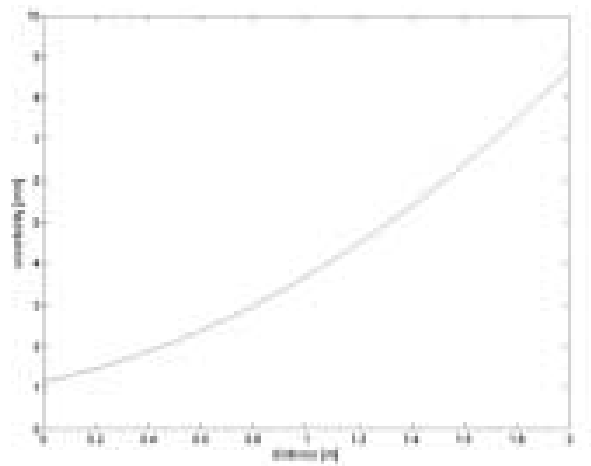

Figure 17: Uncertainty of the single point distance measurement of the laser line striper. The distance is measured form the right side of the vehicle, not the center of the sensor.

For the uncertainty of the curb location alongside the vehicle we have the additional error of the vehicle state estimator, which is much less than $1 \%$ of the distance traveled.

We estimated the uncertainty in the curb location measured with the video by comparing it with the much more precise measurement of the laser line striper. While driving alongside a curb, positions measured by the video will a short time later be also measured by the line striper. We found an average uncertainty of $24 \mathrm{~mm}$ for distances of $0 \mathrm{~m}$ to $3 \mathrm{~m}$ in front of the vehicle, of $45 \mathrm{~mm}$ for $3 \mathrm{~m}$ to $10 \mathrm{~m}$ and up to $250 \mathrm{~mm}$ for the most difficult condition of a turn at a distance of approximately $10 \mathrm{~m}$.

\section{Future Work}

We have shown in this article that we are able to fuse data from a laser line striper, vehicle state estimation, video image, and object detection together to give us reliable and comprehensive measurements of continuous objects alongside the vehicle. Some aspects of the different components can still be improved to eliminate false detections. We intend to incorporate this system on different platforms, e.g. a warning system for transit buses and precision docking.

\section{References}

[1] Aufrère, R., Chapuis, R., and Chausse, F., "A model driven approach for real time road recognition", International Journal of Machine Vision and Applications, December 2001, Vol. 13, p. 93-107.

[2] Broggi, A., "Parallel and Local Feature Extraction: a real-time approach to road boundary detection", IEEE Transactions on Image Processing, Vol. 4, N², p. 217223, 1995.

[3] Canny, J., “A computational Approach to Edge Detection", IEE Transactions on Pattern Analysis and Machine Intelligence, Vol. 8, Nº 6, November 1986.

[4] Dickmanns, E.D., and Mysliwetz, B.D., "Recursive 3D Road and Relative Ego-State Recognition", IEEE Transactions Pattern Analysis and Machine Intelligence, Vol. 14, N², p. 199-213, 1992.

[5] Mertz, C., Kozar, J., Miller, J.R., and Thorpe, C., "Eye-safe Laser Line Striper for Outside Use", IEEE Intelligent Vehicle Symposium, June 2002.

[6] Mertz, C., McNeil, S., Thorpe, C., "Side Collision Warning Systems for Transit Buses" Proceedings of the IEEE Intelligent Vehicle Symposium, October 2000.

[7] McNeil, S., et al., "A Performance Specification for Transit Bus Side Collision Warning System" Proceedings of the 9th World Congress on Intelligent Transport Systems, October 2002.

[8] Pomerleau, D.A., "RALPH: Rapidly Adapting Lateral Position Handler", IEEE Symposium on Intelligent Vehicles, p. 506-511, September 1995.

[9] Thorpe, C., et al., "Dependable Perception for Robots", Proceedings of International Advanced Robotics Program IEEE, Robotics and Automation Society, Seoul, Korea, May 2001.

[10] Thorpe, C., et al., "Driving in Traffic: Short-Range Sensing for Urban Collision Avoidance", Proceedings of SPIE: Unmanned Ground Vehicle Technology IV, Vol. 4715.
The views can conclusions contained in this document are those of the authors and should not be interpreted as representing the official policies, either expressed or implied, of the sponsors or the U.S. Government. 